\title{
Evidence That Deletion of ETS-1, a Gene in the Jacobsen Syndrome (11q-) Cardiac Critical Region, Causes Congenital Heart Defects through Impaired Cardiac Neural Crest Cell Function
}

\author{
Maoqing Ye, Yan Yin, Kazumi Fukatsu, and Paul Grossfeld
}

\begin{abstract}
Jacobsen syndrome (11q-) is a rare chromosomal disorder characterized by multiple problems including congenital heart defects, behavioral problems, intellectual disability, dysmorphic features, and bleeding problems. Septal defects, including double outlet right ventricle (DORV), are among the most common CHDs that occur in 11q-. One possible mechanism underlying the CHDs and other problems in 11q- is a defect in neural crest cell function. The E26 avian leukemia 1, 5' domain (ETS-1) gene is a member of the ETS-domain transcription factor family. ETS-1 is deleted in every 11q- patient with CHDs, and gene-targeted deletion of the ETS-1 gene in C57/B6 mice causes DORV with $100 \%$ penetrance. Normal murine cardiac development requires precisely regulated specification of the cardiac neural crest cells (cNCCs). To begin to define the role of ETS-1 in mammalian cardiac development, we have demonstrated that ETS-1 is strongly expressed in mouse cNCCs during early heart development. Sox10 is a key regulator for the neural crest cell gene regulatory network. It is also an early marker for NCCs, and its expression can facilitate the analysis of cNCC function during embryonic development. We have demonstrated that loss of ETS-1 causes decreased migrating Sox 10expressing cells in E10.5 C57/B6 mouse embryos. These results suggest a NCC migration defect in ETS-1 mutants. Our data support the hypothesis that ETS-1 is required for specification and migration of cNCCs and for regulating a cNCC-specific gene regulatory network that is required for normal cardiac development.
\end{abstract}

M. Ye, M.D., Ph.D. • Y. Yin, Ph.D. • K. Fukatsu, Ph.D. • P. Grossfeld, M.D. ( $\varangle)$

Division of Pediatric Cardiology, Department of Pediatrics, UCSD School of Medicine, La Jolla, CA, USA

e-mail: pgrossfeld@ucsd.edu

T. Nakanishi et al. (eds.), Etiology and Morphogenesis of Congenital Heart Disease, DOI 10.1007/978-4-431-54628-3_52 


\section{Keywords}

Jacobsen syndrome - Cardiac neural crest - ETS-1 • Double outlet right ventricle $\cdot$ Genetic modifier

\subsection{Introduction}

Congenital heart defects (CHDs) are the most common birth defect in live-born infants, occurring in $0.7 \%$ of the general population. Although there are numerous genetically engineered mouse models for CHDs, only a small number of these genes are currently associated with CHDs in humans.

Conotruncal defects (CTDs), including double outlet right ventricle (DORV), are among the most common CHDs in the general population and usually require surgical repair to ensure a normal life expectancy. Little is known about the molecular and cellular mechanisms underlying the development of CTDs in humans. Normal murine cardiac development requires precisely regulated specification of the cardiac neural crest cells (cNCCs) and subsequent migration to the developing outflow tract. In animal models, impairment of NCCs causes CTDs [1].

The 11q terminal deletion disorder (11q-, Jacobsen syndrome) (OMIM \# 1477910) is caused by heterozygous deletions in distal 11q (Fig. 52.1).

Fifty-six percent of patients have CHDs (Table 52.1). Septal defects, including DORV, account for about half of all CHDs that occur in 11q- patients.

As shown in Fig. 52.2, we have identified a Jacobsen syndrome cardiac "critical" region in distal 11q containing only five known genes, including the ETS-1 transcription factor.

The ETS-1 gene is a member of the ETS-domain transcription factor family. ETS factors have important roles in a host of biological functions, including the regulation of cellular growth and differentiation as well as organ development.

\section{$11 q$ terminal deletion disorder}
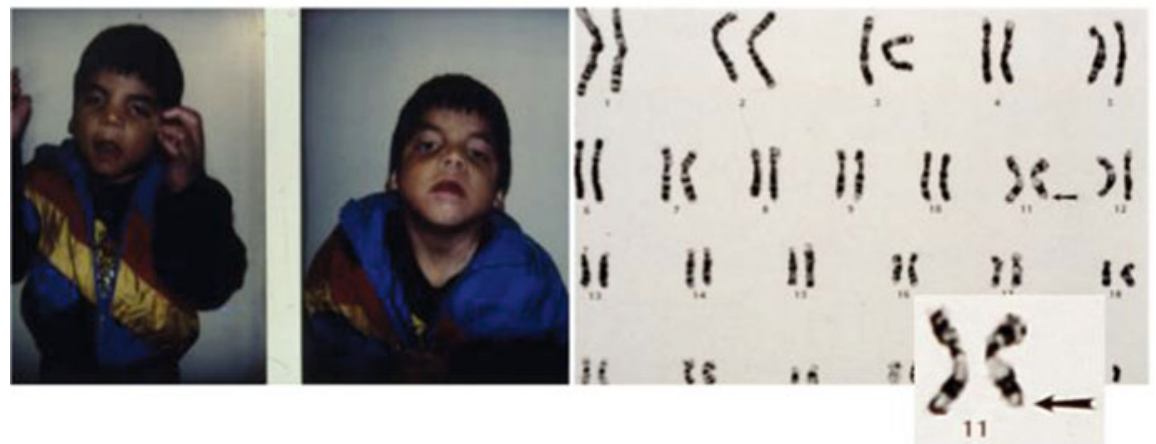

Fig. 52.1 Patient with Jacobsen syndrome. Karyotype demonstrates large terminal deletion in $11 \mathrm{q}$ 
Table 52.1 CHDs in 11q-

\begin{tabular}{l}
\hline Left-sided/flow lesions (two-thirds) \\
\hline Hypoplastic left heart syndrome ${ }^{\mathrm{a}}$ \\
\hline Shone's complex \\
\hline Coarctation \\
\hline Bicuspid aortic valve \\
\hline Aortic valve stenosis \\
\hline Mitral valve stenosis \\
\hline Ventricular septal defect \\
\hline Less common heart defects (one-third) \\
\hline Secundum atrial septal defect \\
\hline Aberrant right subclavian artery \\
\hline Atrioventricular septal canal defect \\
\hline D-transposition of the great arteries \\
\hline Dextrocardia \\
\hline Left-sided superior vena cava \\
\hline Tricuspid atresia \\
\hline Type B interruption of the aortic arch/truncus arteriosus \\
\hline Pulmonary atresia/intact ventricular septum \\
\hline TAPVR \\
\hline Ebstein anomaly \\
\hline Tetralogy of Fallot
\end{tabular}

${ }^{\mathrm{a}} \sim 10 \%$ born with HLHS; $\sim 1-2 \%$ of all HLHS pts

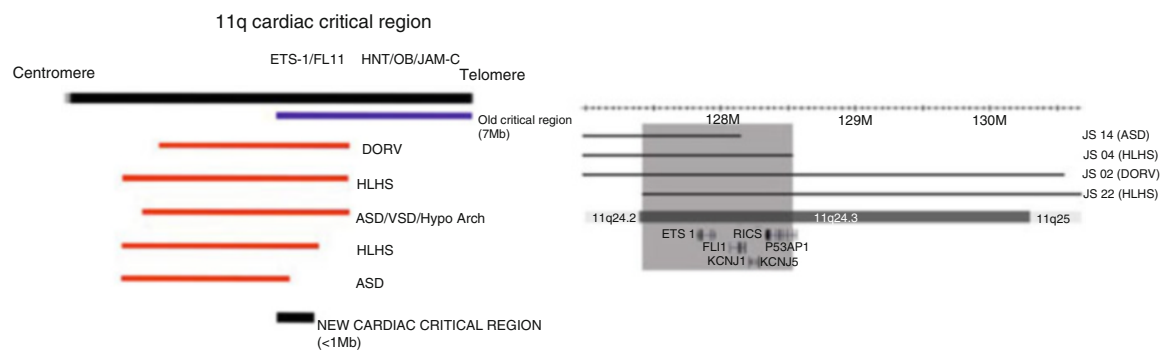

Fig. 52.2 Cardiac "critical" region in 11q, defined by region of overlap between smallest terminal deletion and interstitial deletions in patients with Jacobsen syndrome clinical phenotype, including congenital heart defects

Until recently, nothing was known about the function of ETS-1 in mammalian heart development or its possible role in causing human congenital heart disease.

Although little is known about the mechanisms underlying ETS-1 in mammalian heart development, recent studies in the ascidian Ciona intestinalis have demonstrated that ETS-1 regulates two critical aspects of heart development: heart progenitor cell migration and heart cell differentiation. Interestingly, loss of ETS-1 abolishes normal heart cell migration during development, resulting in an ectopically located heart chamber. Taken together, these results indicate that ETS-1 
is required for normal cell migration in heart development, although the mechanism (s) underlying these cell migration defects remains to be elucidated.

\subsection{Evidence for a Role for ETS-1 in the Cardiac Neural Crest in Mice}

\subsubsection{Expression of ETS-1 in Cardiac Lineages During Murine Heart Development}

To begin to define the role of ETS-1 in mammalian cardiac development, we have performed in situ hybridization studies on mouse embryos and have shown that ETS-1 is strongly expressed in murine cNCCs as well as the endocardium during early heart development (Fig. 52.3).

\subsubsection{ETS-1 Mutant Mice Have a Double Outlet Right Ventricle (DORV) Phenotype}

To determine if loss of ETS-1 causes congenital heart defects, we have analyzed gene-targeted ETS-1 deletion mice. As shown in Fig. 52.4, ETS-1 homozygous null mice in a C57/B6 background exhibit DORV with $100 \%$ penetrance, resulting in perinatal lethality [2].

\subsubsection{Lost of ETS-1 Causes Decreased Expression of Sox10}

We have previously demonstrated that ETS-1 expression is expressed in cNCCs and endocardium during murine embryonic development. Sox10 is a key regulator in the NCC gene regulatory network. It is critical for migration and specification of NCC fate. To examine the role of ETS-1 in murine cardiac NCC migration, we examined Sox 10 expression in ETS-1-/- mutant and control C57/B6 embryos at E10.5 by using whole-mount in situ hybridization analysis. Expression of Sox 10 in the NCCs in the pharyngeal arch region and dorsal root ganglia was reduced in ETS-1-/- mutant embryos, suggestive of a cNCC migration defect as shown in Fig. 52.5 (left). The result was confirmed independently by quantitative RT-PCR analysis (right).

\subsection{Establishment of an Explanted cNCC "Ex Vivo" Culture System}

We hypothesized that defects in cNCCs migration should be able to be reproduced in an "ex vivo" culture system. Toward that end, we have utilized an explanted culture system to observe cNCC migration [3]. Mouse embryos were collected from 

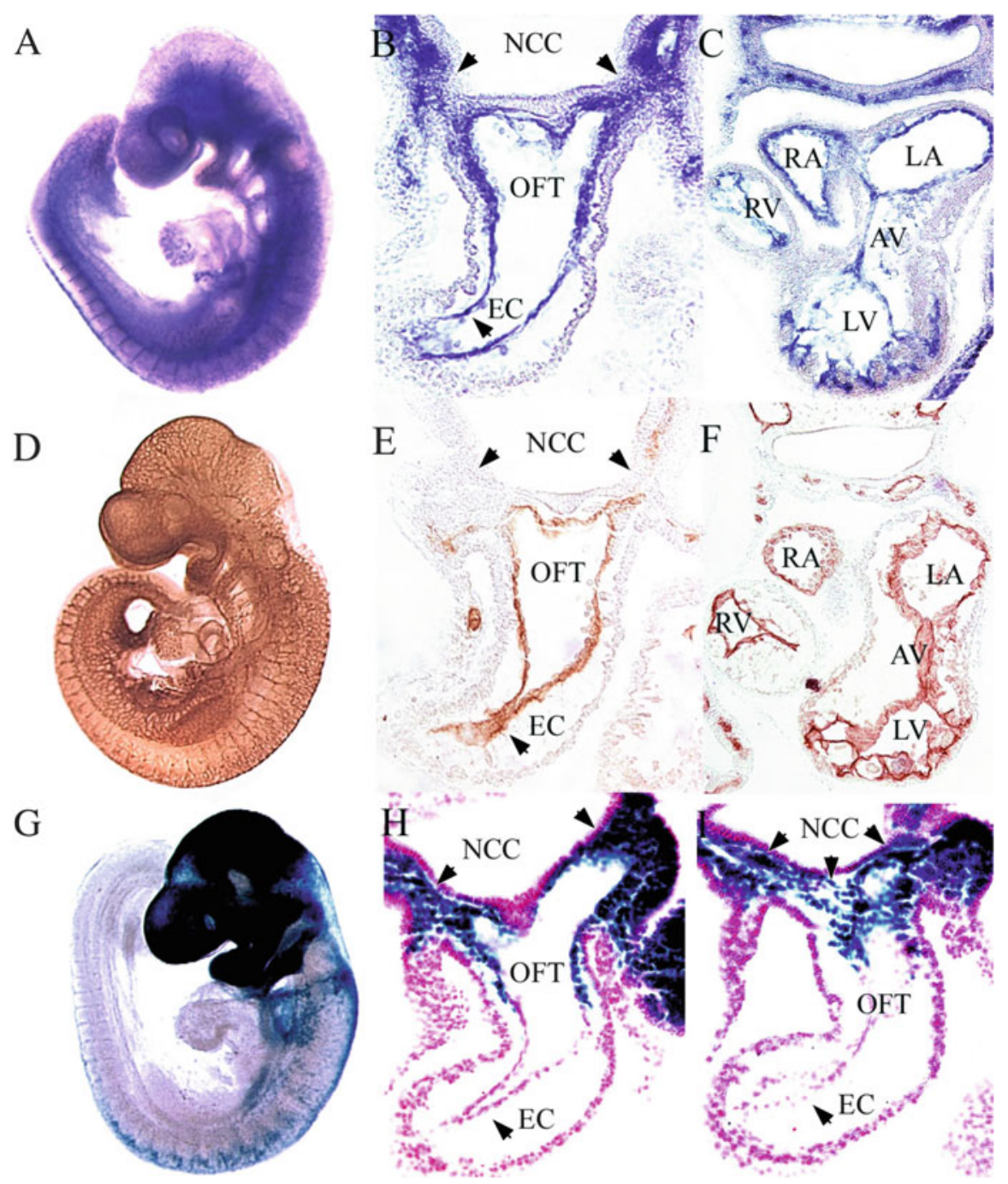

Fig. 52.3 Expression of ETS-1 in the heart in ED9.5 embryos: in situ hybridizations are shown in (a) (whole mount) and in sections (b) (anterior coronal section) and (c) (posterior coronal section). Immunohistochemistry indicating endothelial expression using a PECAM (CD31) antibody is shown in (d) (whole mount) and in sections (e) (anterior coronal) and (f) (posterior coronal). LacZ staining of neural crest using a Wnt1-Cre; ROSA26 LacZ indicator strain is shown in (g) (whole mount), (h) (anterior coronal), and (i) (posterior coronal)

C57/B6 background embryos at E8.5, coinciding with the onset of cNCC migration toward the heart. E8.5 embryos were collected and treated by dispase to dissociate the tissue gently. After treatment, neural tubes from somite one to three region (cardiac neural crest) were dissected out and cut into $100 \times 300$ um pieces. Each 


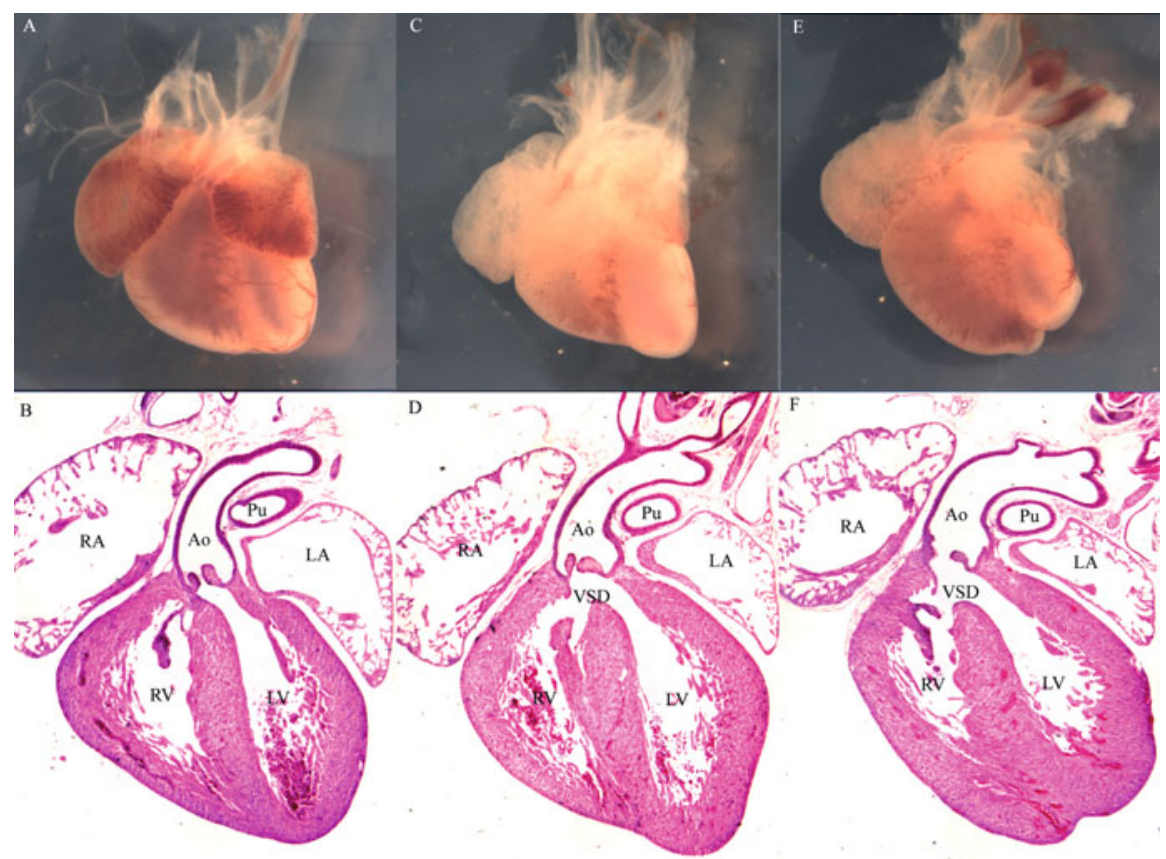

Fig. 52.4 Gene-targeted knockout of ETS-1 in C57/B6 E16.5 mice, showing double outlet right ventricle with normally related great arteries. Wild type is shown in (a) and (b); two mutant hearts are shown in panels (c-f). $R A$ right atrium, $L A$ left atrium, $R V$ right ventricle, $L V$ left ventricle, $P u$ pulmonary artery, $A o$ aorta, $V S D$ ventricular septal defect

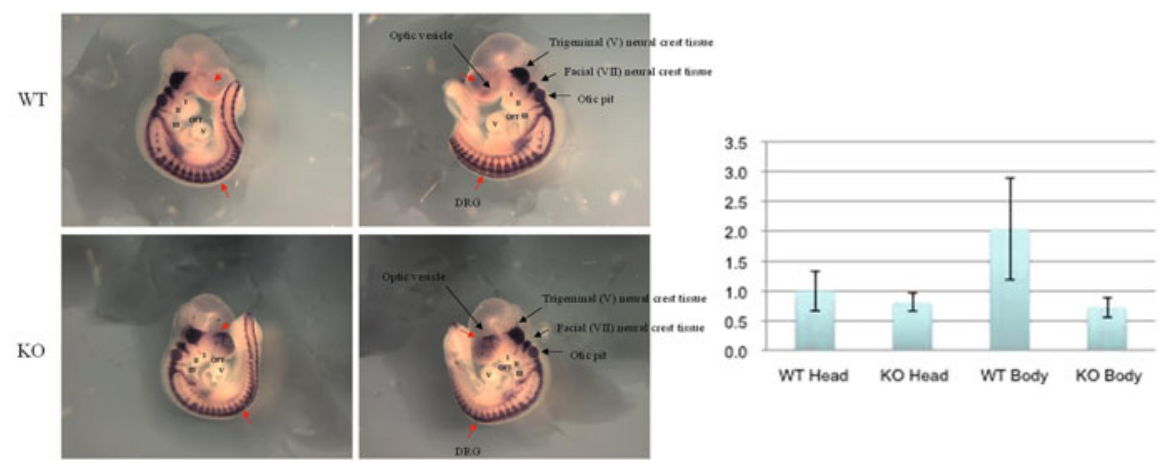

Fig. 52.5 Whole mount study demonstrating decreased Sox 10 expression in E10.5 ETS-1-/C57/B6 embryos (left, lower panel) compared to wild type (left, upper panel). Real-time quantitative PCR demonstrates decreased Sox 10 expression in the body of E10.5 embryos in ETS-1-/embryos, compared to wild type (right) 
piece was placed on fibronectin-coated glass bottom slides and incubated in culture media in $5 \% \mathrm{CO}_{2}$ and $21 \% \mathrm{O}_{2}$. After $24 \mathrm{~h}$ incubation, we performed DAPI staining and obtained images of the migrating cells. Representative results from WT and ETS-1 ${ }^{-1-}$ mice are shown in Fig. 52.6.

\subsubsection{Loss of ETS-1 in C57/B6 Mice Causes Decreased NCC Numbers and Decreased Migration}

To analyze the migration distance using our ex vivo system, we counted the number of migrating cells in each explanted culture. The migration distance was divided into three distanced from the neural tube edge: $0-150 \mu \mathrm{m}, 150-300 \mu \mathrm{m}$, and over $300 \mu \mathrm{m}$ edge. The total number of cells that had migrated for each distance was manually counted, and the percentage of the total for each migration distance was determined. As shown in Fig. 52.6, cNCCs from ETS-1-/- C57/B6 embryos were fewer in number and had decreased migration distance. The percentage of total cells migrating $>300 \mu \mathrm{m} 24 \mathrm{~h}$ after explantation in ETS-1-/- mutant embryos is fourfold lower than control. The cell number per embryo was also significantly decreased in ETS-1-/- mutants.
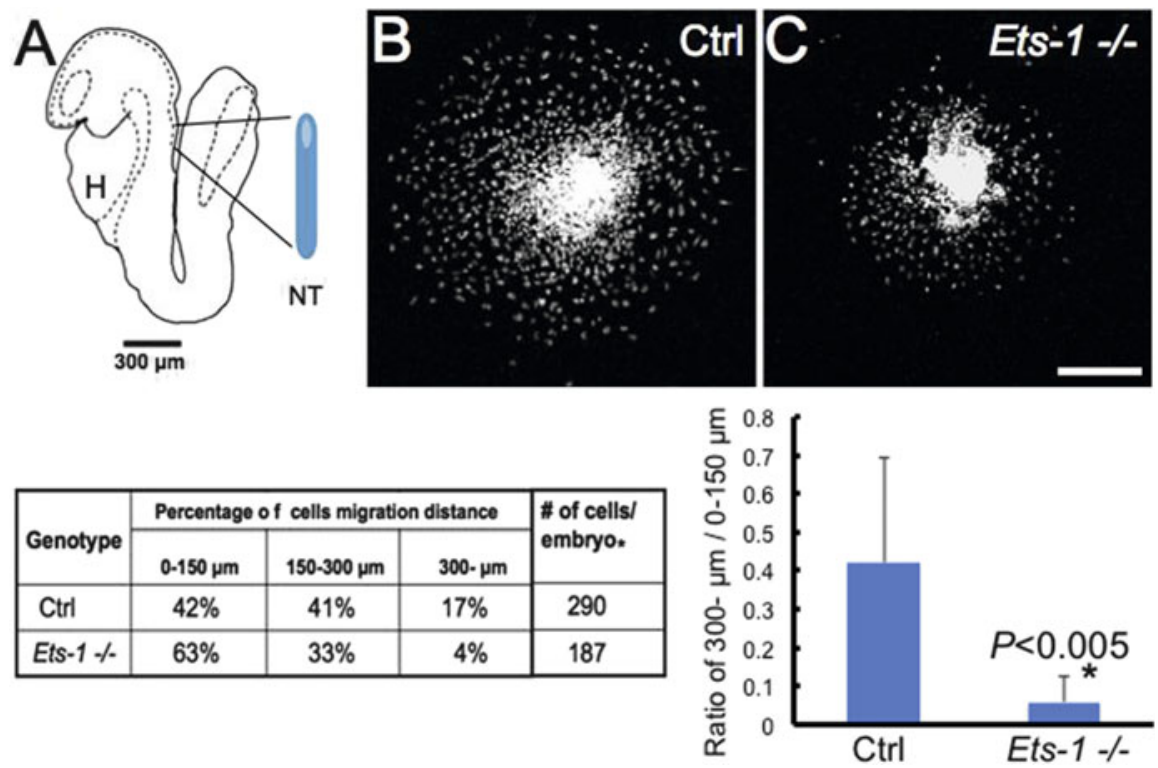

Fig. 52.6 Cardiac neural crest cells migration in explant culture. (A) Schematic of E8.5 embryo. Blue bar shows the neural tubes somite one to three region. $H$ heart, $N T$ neural tube. $(B)$ Representative images with DAPI staining of $24 \mathrm{~h}$ cultured cardiac neural crest cells in control $(B)$ and ETS-1-/- mutants $(C)$. Scale bar, $300 \mu \mathrm{m}$. Both images were taken by an inverted confocal microscope FV-1000 using a $10 \times$ objective lens. $(D)$ Quantification of migration distances, demonstrating impaired migration ability in ETS-1-/- cells compared to wild type 
We then calculated the ratio of $300+\mu \mathrm{m}$ over $0-150 \mu \mathrm{m}$ of cultured migration cells. As shown in Fig. 52.6, the ratio was significantly reduced in ETS-1-/mutants.

\subsection{Cardiac Neural Crest Cell Number and Migration Are Preserved in ETS-1- $I-$ Mice in an FVBN-1 Background}

The cardiac phenotype in ETS-1 knockout mice is dependent on the genetic background. In contrast to C57/B6 mice, ETS-1-/- mutant mice in an FVBN-1 background have normal hearts. Consistent with a neural crest cell autonomous mechanism for causing DORV in the C57/B6 strain, ex vivo studies demonstrate normal cNCC numbers and migration in ETS-1-/- FVBN-1 embryos (data not shown).

\subsection{Summary, Future Directions, and Clinical Implications}

Using human and murine genetics systems, we have identified the ETS-1 transcription factor gene as the likely causative gene for CHDs in Jacobsen syndrome. Our expression data implicate an important role for ETS-1 in the cardiac neural crest during murine heart development. Based on the known function of the ETS-1 homologue in the ascidian Ciona intestinalis and using an ex vivo cell migration system, we hypothesize that ETS- 1 is essential for early cNCC fate determination and migration in mammalian heart development. Future studies will include performing in vivo real-time imaging and lineage fate mapping studies in the neural crest to delineate how loss of ETS- 1 causes decreased cNCCs in the developing heart [4], whether there is a NCC-autonomous mechanism and whether there is a migration defect. Importantly, loss of ETS-1 in FVBN-1 mice does not cause congenital heart defects, suggesting the presence of a genetic modifier(s) that can prevent the development of CHDs in the absence of ETS-1. To address this, determination of a neural crest cell autonomous mechanism would implicate a neural crest cell-specific modifier. Identification of such a genetic modifier could have important implications for the prevention of certain congenital heart defects.

Open Access This chapter is distributed under the terms of the Creative Commons AttributionNoncommercial 2.5 License (http://creativecommons.org/licenses/by-nc/2.5/) which permits any noncommercial use, distribution, and reproduction in any medium, provided the original author(s) and source are credited.

The images or other third party material in this chapter are included in the work's Creative Commons license, unless indicated otherwise in the credit line; if such material is not included in the work's Creative Commons license and the respective action is not permitted by statutory regulation, users will need to obtain permission from the license holder to duplicate, adapt or reproduce the material. 


\section{References}

1. Scholl AM, Kirby M. Signals controlling neural crest contributions to the heart. Wiley Interdiscip Rev Syst Biol Med. 2009 April 29;1(2):220-7. Srivastava D. Genetic regulation of cardiogenesis and congenital heart disease. Annu Rev Pathol. 2006;1:199-21.

2. Ye M, Coldren C, Benson W, Goldmuntz E, Ostrowski M, Watson D, Perryman B, Grossfeld P. Deletion of ETS-1, a gene in the Jacobsen syndrome critical region, causes ventricular septal defects and abnormal ventricular morphology in mice. Hum Mol Genet. 2010;19(4):648-56.

3. Epstein JA, Li J, Lang D, Chen F, Brown CB, Jin F, Lu MM, Thomas M, Liu E, Wessels A, Lo CW. Migration of cardiac neural crest cells in Splotch embryos. Development. 2000;127 (9):1869-78.

4. Gao Z, Kim GH, Mackinnon AC, Flagg AE, Bassett B, Earley JU, Svensson EC. Ets1 is required for proper migration and differentiation of the cardiac neural crest. Development. 2010;137(9):1543-5124. 